Research Article

\title{
Lower Extremity Skeletal Muscle Mass, but Not Upper Extremity Skeletal Muscle Mass, Is Inversely Associated with Hospitalization in Patients with Type 2 Diabetes
}

\author{
Hidetaka Hamasaki ${ }^{1,2}$ \\ ${ }^{1}$ Hamasaki Clinic, Kagoshima, Japan \\ ${ }^{2}$ Department of Internal Medicine, Kohnodai Hospital, National Center for Global Health and Medicine, Chiba, Japan \\ Correspondence should be addressed to Hidetaka Hamasaki; hhamasaki78@gmail.com
}

Received 14 June 2017; Accepted 30 July 2017; Published 7 August 2017

Academic Editor: Maria Pia Francescato

Copyright ( 2017 Hidetaka Hamasaki. This is an open access article distributed under the Creative Commons Attribution License, which permits unrestricted use, distribution, and reproduction in any medium, provided the original work is properly cited.

\begin{abstract}
Aim. To investigate the association of skeletal muscle mass with metabolic parameters and hospitalization in patients with type 2 diabetes. Methods. A retrospective observational study was conducted in patients with type 2 diabetes between May 2013 and November 2015. Body composition was measured by bioelectrical impedance analysis. Multiple regression analysis was performed to identify the association between skeletal muscle mass and metabolic parameters. Cox proportional hazard analysis was performed to assess the association between skeletal muscle mass and hospitalization. Results. A total of 121 patients were enrolled in this study. The mean age of patients was $59.4 \pm 14.2$ years. During a mean follow-up of $730 \pm 253$ days, three patients (2.8\%) died and 79 patients $(65.3 \%)$ were admitted to our hospital. After adjustment for age, sex, height, and weight, it was found that lower extremity skeletal muscle mass (LSM) was inversely associated with brachial-ankle pulse wave velocity $(\beta=-0.108, P=0.008)$. Moreover, LSM was significantly associated with reduced risk of hospitalization (hazard ratio $=0.752 ; 95 \%$ confidence interval, $0.601-0.942 ; P=0.013$ ). In contrast, upper extremity skeletal muscle mass (USM) did not exhibit any significant association. Conclusion. LSM, but not USM, is important for managing patients with type 2 diabetes. This trial is registered with UMIN000023010.
\end{abstract}

\section{Introduction}

Decreased strength and impaired function of skeletal muscle are associated with increased mortality [1-3]. Sarcopenia, an age-related decline in muscle mass and function, has a negative impact on quality of life, disability, and mortality in older individuals [4]; thus, prevention of sarcopenia is important. Patients with type 2 diabetes show lower muscle strength [5] than those without type 2 diabetes. A previous study showed that type 2 diabetes is associated with skeletal muscle loss in community-dwelling older individuals [6]. Current evidence suggests that patients with type 2 diabetes have reduced muscle strength and impaired physical function due to hyperglycemia, insulin resistance, ectopic fat in skeletal muscle, and progressed diabetic neuropathy [7]. We have previously reported that lower extremity skeletal muscle mass (LSM) is important for improving metabolic parameters and body composition in obese patients with type 2 diabetes $[8,9]$. Moreover, Frank-Wilson et al. [10] showed that lower extremity muscle density is associated with fall risk in older individuals. Guadalupe-Grau et al. [11] have also reported that the maximal isometric strength of knee and hip muscle is a predictor of mortality and hospitalization in women. However, no longitudinal studies have investigated the association between LSM and disease prognosis in patients with type 2 diabetes. The author hypothesizes that LSM plays an important role in managing type 2 diabetes. Hence, the aim of this study was to examine the association of LSM with hospitalization in Japanese patients with type 2 diabetes. 


\section{Methods}

2.1. Study Design and Patients. A retrospective cohort study was conducted in patients with type 2 diabetes who were treated at the Kohnodai Hospital, National Center for Global Health and Medicine, Japan between May 2013 and November 2015. A total of 121 patients whose body composition was measured at the outpatient clinic were included. Patients were instructed to consume a calorierestricted diet of $25-30 \mathrm{kcal} / \mathrm{kg}$ (ideal body weight) per day, which was prescribed by certified nutritional educators as diet therapy for diabetes, during the study period. All patients were evaluated and followed until death or till the end of follow-up in May 2016. General information, such as date of hospital admission, duration of hospitalization, and cause of hospitalization, was retrieved from the electronic health record of our hospital. The study protocol was approved by the Medical Ethics Committee of the National Center for Global Health and Medicine (reference number NCGM-G-002052), and the study was performed in accordance with the Declaration of Helsinki. The opt-out method of obtaining informed consent was used. The patients were anonymized to protect their personal information.

2.2. Anthropometric and Physiological Measurements. Patients' height and weight were measured using a rigid stadiometer (TTM stadiometer; Tsutsumi Co. Ltd., Tokyo, Japan) and calibrated scales (AD-6107NW; A\&D Medical Co. Ltd., Tokyo, Japan), respectively. Body mass index (BMI) was calculated as body weight in kilograms divided by the square of body height in meters. Waist circumference was measured at the umbilical level at the end of exhalation in standing position. Blood pressure was measured in sitting position using an automatic sphygmomanometer (HBP9020; Omron Co. Ltd., Tokyo, Japan). Arterial stiffness was examined by measuring both brachial-ankle pulse wave velocity (baPWV) and heart rate-corrected augmentation index $\left(\mathrm{AIx}_{75}\right)$ using a pulse pressure analyzer (BP-203RPE; Nihon Colin, Tokyo, Japan) and a digital automatic sphygmomanometer (HEM-9000AI; Omron Co. Ltd., Tokyo, Japan), respectively. The average value of right and left baPWV was used for the analysis.

2.3. Analyses of Lifestyle and Medical History. Technicians from the Clinical Research Center of the National Center for Global Health and Medicine at Kohnodai Hospital asked patients about their duration of diabetes, smoking status, alcohol consumption, physical activity levels, and history of cardiovascular disease (CVD), such as stroke, nonfatal coronary heart disease, or peripheral artery disease, at the outpatient clinic. To quantify patients' smoking status, the Brinkman index (number of cigarettes per day multiplied by the number of years) was calculated [12]. Patients were also asked about their regular exercise habits using a simple questionnaire. The duration of exercise performed per day was calculated using a physical activity questionnaire, which comprised three items: (1) Do you engage in any physical exercise? If yes, what kind of exercise do you perform regularly?; (2) How often do you exercise in a week?; and (3) How long do you exercise per session?

2.4. Blood Examination. Blood samples were taken from the antecubital vein in the morning. Plasma glucose and hemoglobin Alc (HbAlc) levels were measured. Enzymatic methods were used to assess plasma glucose (Glucose Assay Kit; Wako Pure Chemical Industries, Osaka, Japan). HbA1c levels were measured using automated enzyme-linked immunosorbent assays (E-test TOSOH II; Tosoh, Tokyo, Japan).

2.5. Analysis of Body Composition. Body composition was analyzed using a bioelectrical impedance analysis device (InBody720; Biospace Co. Ltd, Tokyo, Japan), which uses a patented 8-point tactile electrode system. This method is based on the principle that lean body mass contains higher water and electrolyte content than fatty tissue; hence, these tissues can be distinguished by electrical impedance. The bioelectrical impedance analysis device uses six frequencies $(1,5$, $50,250,500$, and $1000 \mathrm{kHz}$ ) to produce 30 impedance values for five body segments, such as right upper extremity, left upper extremity, trunk of the body, right lower extremity, and left lower extremity [13]. A previous study concerning validation of this method has demonstrated that body composition measured using this device is highly correlated with dual-energy X-ray absorptiometry measurements [14]. Body composition analysis was performed within 3 months after acquiring medical history. Upper extremity skeletal muscle mass (USM) and LSM were calculated by summing right and left upper and lower extremity skeletal muscle mass, respectively.

2.6. Statistical Analysis. Statistical analyses were performed using SPSS version 24 (IBM Co. Ltd., Chicago, IL, U.S.). All values are expressed as mean \pm standard deviation, except for sex. Pearson's correlation coefficient was calculated to analyze the correlation of USM and LSM with clinical data. In addition, multiple regression analysis adjusted for confounding covariates such as age, sex, height, and weight was performed to test the independent association of USM and LSM with clinical parameters. Furthermore, Cox proportional hazards analysis was performed to assess the independent association of USM and LSM with hospitalization. We included age, sex, height, weight, history of CVD, smoking status (Brinkman index), alcohol consumption, exercise time, HbAlc levels, and duration of diabetes in the Cox model. The entry variable was the date of acquiring medical history at the outpatient clinic. Follow-up was at the first day of hospitalization or May 1,2016 , whichever came first. $P$ values $<0.05$ were considered statistically significant.

\section{Results}

A total of 121 patients (67 men and 54 women) were enrolled in the present study. The mean age of patients was $59.4 \pm 14.2$ years, and mean BMI was $27.6 \pm 6.8 \mathrm{~kg} / \mathrm{m}^{2}$. Table 1 shows clinical characteristics of patients. Total skeletal muscle mass, USM, and LSM were inversely correlated with $\mathrm{AIx}_{75}(r=-0.402, \quad P=0.001 ; \quad r=-0.317, \quad P=0.008 ;$ and 
TABLE 1: Characteristics of study subjects.

\begin{tabular}{|c|c|}
\hline \multicolumn{2}{|l|}{ Demographics } \\
\hline$n$ & 121 \\
\hline Age (years) & $59.4(14.2)$ \\
\hline Sex (men/women) & $67 / 54$ \\
\hline Alcohol consumption (g ethanol per day) & $21.9(39.4)$ \\
\hline Brinkman index & $275.9(483.6)$ \\
\hline Exercise time (min/day) & $14.6(41.4)$ \\
\hline History of cardiovascular disease (yes/no) & $7 / 114$ \\
\hline Duration of diabetes (years) & $7.9(9.3)$ \\
\hline \multicolumn{2}{|l|}{ Anthropometric data } \\
\hline Height $(\mathrm{cm})$ & $161.7(10.4)$ \\
\hline Weight (kg) & $72.4(20.7)$ \\
\hline BMI $\left(\mathrm{kg} / \mathrm{m}^{2}\right)$ & $27.6(6.8)$ \\
\hline Waist circumference $(\mathrm{cm})$ & $95.9(16.1)$ \\
\hline \multicolumn{2}{|l|}{ Body composition } \\
\hline Total skeletal muscle mass (kg) & $25.3(6.3)$ \\
\hline Upper extremity skeletal muscle mass (kg) & $5.0(1.6)$ \\
\hline Lower extremity skeletal muscle mass (kg) & $14.4(3.9)$ \\
\hline Body fat mass (kg) & $24.3(12.9)$ \\
\hline Body fat percentage (\%) & $32.6(10.9)$ \\
\hline \multicolumn{2}{|l|}{ Physiological and biochemical data } \\
\hline Systolic blood pressure (mmHg) & $138.9(22.9)$ \\
\hline Diastolic blood pressure (mmHg) & $79.8(14.3)$ \\
\hline Plasma glucose $(\mathrm{mg} / \mathrm{dL})$ & $178.3(78.4)$ \\
\hline $\operatorname{HbA1c}(\%)$ & $8.5(2.1)$ \\
\hline Brachial-ankle pulse wave velocity $(\mathrm{cm} / \mathrm{s})$ & $1697(451)$ \\
\hline Augmentation index & $74.3(14.2)$ \\
\hline
\end{tabular}

Data are represented as the mean (SD) except for the number of subjects, sex, and history of cardiovascular disease. BMI: body mass index; HbAlc: hemoglobin A1c.

$r=-0.447, P<0.001$, resp.). Similarly, total skeletal muscle mass and LSM were also inversely correlated with baPWV $(r=-0.239, P=0.022$ and $r=-0.262, P=0.012$, resp.). After adjustment for age, sex, height, and weight, LSM was still inversely associated with baPWV $(\beta=-0.108$, $P=0.008$ ); however, such correlations disappeared after further adjustment for age, sex, height, weight, mean blood pressure, and HbAlc levels. No other significant associations between LSM or USM and clinical parameters were observed. During the mean follow-up of $730 \pm 253$ days, three patients $(2.8 \%)$ died and 79 patients $(65.3 \%)$ were admitted to our hospital. Reasons for hospitalization included glycemic control $(n=63)$, gastroenterological disease $(n=5)$, infectious disease $(n=5)$, hepatic disease $(n=2)$, psychoneurological disease $(n=2)$, respiratory disease $(n=1)$, and ocular disease $(n=1)$. Cox proportional hazards analyses adjusted for age, sex, height, weight, history of CVD, Brinkman index, alcohol consumption, exercise time, mean blood pressure, $\mathrm{HbAlc}$ levels, and duration of diabetes revealed that LSM had a significant association with hospitalization; however, USM had no such association (Table 2).

\section{Discussion}

The present study demonstrates that LSM is associated with reduced risk of hospitalization. To the best of my knowledge, this is the first study to show that LSM has beneficial impact on hospitalization in patients with type 2 diabetes.

Epidemiological studies have shown that higher muscle mass is associated with reduced risk of cardiovascular and all-cause mortality in elderly population [15] and patients with CVD [16]. Heitmann and Frederiksen [17] also showed that a small thigh circumference is related to an increased risk of developing heart disease and total mortality, suggesting that LSM is particularly important for good health. Although the mechanism underlying the association between LSM and mortality has not been clarified, authors have hypothesized that individuals with decreased LSM are sedentary. Physical inactivity promotes skeletal muscle loss; compared with sedentary individuals, physically active individuals seem to retain their leg strength in old age [18]. Indeed, nonexercise activity thermogenesis besides regular exercise is positively correlated with LSM [8]. Moreover, physical inactivity increases the risk of major noncommunicable diseases, including type 2 diabetes [19]. Locomotive physical activity plays a pivotal role in glycemic control and reduction of mortality risk in patients with type 2 diabetes [20]. The association between LSM and risk of hospitalization suggests that LSM is a prognostic marker of health in patients with type 2 diabetes.

Recently, skeletal muscle has been identified as an endocrine organ, which secrets myokines that communicate with adipose tissue, the liver, the pancreas, the bones, and the brain [21]. Myokines include various bioactive peptides such as IL-6, IL-8, IL-15, fibroblast growth factor-21, brain-derived neurotrophic factor, and irisin; some of them have a potential to improve insulin sensitivity, glucose uptake, and glucose oxidation [22]. In this study, most patients were hospitalized because of hyperglycemia. Skeletal muscle loss may deteriorate glycemic control mediated by myokines. However, to elucidate the effect of skeletal muscle mass on glycemic control, further studies are needed in the future.

In addition, skeletal muscle loss is associated with arterial stiffness, which may result in increased risk of CVD or mortality. Ochi et al. [23] have reported that baPWV is an independent risk factor of sarcopenia after evaluating mid-thigh muscle cross-sectional area in middle-aged to elderly men. Lee et al. [24] have also reported that limb muscle mass measured by bioelectrical impedance analysis is inversely associated with augmentation index in Asian population. Skeletal muscle loss causes insulin resistance and physical inactivity [25]. Similarly, arterial stiffness is closely linked to reduced exercise capacity [26]. Therefore, it is clear that the relationship between skeletal muscle mass, physical activity, and vascular elasticity certainly plays a role in maintaining good health.

Several limitations associated with this study need to be addressed. First, this study could not evaluate the association of skeletal muscle mass with mortality or cardiovascular events. Further investigations with a larger number of 
TABLE 2: Cox proportional hazard analysis for evaluating the associations of upper and lower extremity skeletal muscle mass with hospitalization in patients with type 2 diabetes.

\begin{tabular}{|c|c|c|c|c|c|c|}
\hline & \multicolumn{3}{|c|}{ Upper extremity } & \multicolumn{3}{|c|}{ Lower extremity } \\
\hline & HR & $95 \%$ CI & $P$ & $\mathrm{HR}$ & $95 \% \mathrm{CI}$ & $P$ \\
\hline Age (per 1 year increase) & 1.013 & $0.989-1.037$ & 0.29 & 1.010 & $0.987-1.033$ & 0.39 \\
\hline \multicolumn{7}{|l|}{ Sex } \\
\hline Men & 0.769 & $0.322-1.838$ & 0.77 & 0.749 & $0.339-1.652$ & 0.47 \\
\hline Women & (ref) & & & (ref) & & \\
\hline Height (per $1 \mathrm{~cm}$ increase) & 1.015 & $0.967-1.065$ & 0.55 & 1.068 & $1.001-1.139$ & 0.047 \\
\hline Weight (per $1 \mathrm{~kg}$ increase) & 1.020 & $0.990-1.051$ & 0.19 & 1.030 & $1.005-1.055$ & 0.017 \\
\hline Smoking (per 1 unit increase in Brinkman index) & 1.000 & $1.000-1.001$ & 0.35 & 1.000 & $1.000-1.001$ & 0.39 \\
\hline Alcohol consumption (per $1 \mathrm{~g} /$ day increase in ethanol consumption) & 1.121 & $1.001-1.255$ & 0.049 & 1.110 & $0.990-1.244$ & 0.073 \\
\hline \multicolumn{7}{|l|}{ History of CVD } \\
\hline Yes & 1.119 & $0.459-2.728$ & 0.8 & 0.835 & $0.330-2.117$ & 0.71 \\
\hline No & (ref) & & & (ref) & & \\
\hline Exercise time (per $1 \mathrm{~min} /$ day increase) & 0.994 & 0985-1.004 & 0.25 & 0.995 & $0.986-1.004$ & 0.29 \\
\hline Duration of diabetes (per 1 year increase) & 1.015 & $0.984-1.046$ & 0.35 & 1.023 & $0.996-1.050$ & 0.098 \\
\hline Mean blood pressure (per $1 \mathrm{mmHg}$ increase) & 0.999 & $0.986-1.011$ & 0.82 & 0.998 & $0.985-1.010$ & 0.71 \\
\hline HbAlc (per 1\% increase) & 1.348 & $1.181-1.539$ & $<0.001$ & 1.364 & $1.193-1.561$ & $<0.001$ \\
\hline Skeletal muscle mass (per $1 \mathrm{~kg}$ increase) & 0.744 & $0.438-1.263$ & 0.27 & 0.752 & $0.601-0.942$ & 0.013 \\
\hline
\end{tabular}

HR: hazard ratio; CI: confidence interval; HbAlc: hemoglobin Alc.

patients and longer duration are warranted. Second, although the bioelectrical impedance analysis device used in this study was validated, skeletal muscle mass measured using regression equation might have been influenced by measurement conditions. Third, muscle strength of extremities was not measured in this study. However, a significant positive correlation between muscle mass and strength is well established $[27,28]$. Despite these limitations, the present study demonstrates that LSM is important to prevent hospitalization in patients with type 2 diabetes. To ensure this association and assess the effect of LSM on disease prognosis, additional prospective studies are required.

\section{Conclusion}

In conclusion, findings of this study suggest that LSM, but not USM, is essential for good health of patients with type 2 diabetes. Prevention of skeletal muscle loss is important for improving quality of life, disability, and mortality. Leg exercises such as resistance training plus regular walking should be performed to improve LSM in patients with type 2 diabetes. Clinicians should probably pay attention to lower extremity skeletal muscle as well as weight, waist circumference, and BMI for effectively managing type 2 diabetes.

\section{Conflicts of Interest}

No potential conflict of interests relevant to this paper was reported.

\section{Authors' Contributions}

Hidetaka Hamasaki conceived, designed, and performed the study; interpreted the results; and wrote the paper.

\section{Acknowledgments}

The author appreciates the support of the Clinical Research Center, Kohnodai Hospital, National Center for Global Health and Medicine.

\section{References}

[1] F. B. Ortega, K. Silventoinen, P. Tynelius, and F. Rasmussen, "Muscular strength in male adolescents and premature death: cohort study of one million participants," British Medical Journal, vol. 345, article e7279, 2012.

[2] D. P. Leong, K. K. Teo, S. Rangarajan et al., "Prognostic value of grip strength: findings from the prospective urban rural epidemiology (PURE) study," Lancet, vol. 386, no. 9990, pp. 266-273, 2015.

[3] B. Roshanravan, K. V. Patel, L. F. Fried et al., "Association of muscle endurance, fatigability, and strength with functional limitation and mortality in the health aging and body composition study," The Journals of Gerontology. Series A, Biological Sciences and Medical Sciences, vol. 72, no. 2, pp. 284-291, 2017.

[4] E. Marzetti, R. Calvani, M. Tosato et al., "Sarcopenia: an overview," Aging Clinical and Experimental Research, vol. 29, no. 1, pp. 11-17, 2017.

[5] E. Cetinus, M. A. Buyukbese, M. Uzel, H. Ekerbicer, and A. Karaoguz, "Hand grip strength in patients with type 2 diabetes mellitus," Diabetes Research and Clinical Practice, vol. 70, no. 3, pp. 278-286, 2015.

[6] S. W. Park, B. H. Goodpaster, J. S. Lee et al., "Excessive loss of skeletal muscle mass in older adults with type 2 diabetes," Diabetes Care, vol. 32, no. 11, pp. 1993-1997, 2009.

[7] L. Bianchi and S. Volpato, "Muscle dysfunction in type 2 diabetes: a major threat to patient's mobility and independence," Acta Diabetologica, vol. 53, no. 6, pp. 879-889, 2016. 
[8] H. Hamasaki, Y. Kawashima, H. Adachi et al., "Associations between lower extremity muscle mass and metabolic parameters related to obesity in Japanese obese patients with type 2 diabetes," PeerJ, vol. 3, article e942, 2015.

[9] H. Hamasaki, Y. Kawashima, Y. Tamada et al., "Associations of low-intensity resistance training with body composition and lipid profile in obese patients with type 2 diabetes," PLoS One, vol. 10, no. 7, article e0132959, 2015.

[10] A. W. Frank-Wilson, J. P. Farthing, P. D. Chilibeck et al., "Lower leg muscle density is independently associated with fall status in community-dwelling older adults," Osteoporosis International, vol. 27, no. 7, pp. 2231-2240, 2016.

[11] A. Guadalupe-Grau, J. A. Carnicero, A. Gómez-Cabello et al., "Association of regional muscle strength with mortality and hospitalisation in older people," Age and Ageing, vol. 44, no. 5, pp. 790-795, 2015.

[12] G. L. Brinkman and E. O. Coates Jr., "The effect of bronchitis, smoking, and occupation on ventilation," The American Review of Respiratory Disease, vol. 87, pp. 684-693, 1963.

[13] L. J. Anderson, D. N. Erceg, and E. T. Schroeder, "Utility of multifrequency bioelectrical impedance compared with dualenergy $\mathrm{x}$-ray absorptiometry for assessment of total and regional body composition varies between men and women," Nutrition Research, vol. 32, no. 7, pp. 479-485, 2012.

[14] A. L. Gibson, J. C. Holmes, R. L. Desautels, L. B. Edmonds, and L. Nuudi, "Ability of new octapolar bioimpedance spectroscopy analyzers to predict 4-componentmodel percentage body fat in Hispanic, black, and white adults," The American Journal of Clinical Nutrition, vol. 87, no. 2, pp. 332-338, 2008.

[15] A. Spahillari, K. J. Mukamal, C. DeFilippi et al., "The association of lean and fat mass with all-cause mortality in older adults: the cardiovascular health study," Nutrition, Metabolism, and Cardiovascular Diseases, vol. 26, no. 11, pp. 10391047, 2016.

[16] P. Srikanthan, T. B. Horwich, and C. H. Tseng, "Relation of muscle mass and fat mass to cardiovascular disease mortality," The American Journal of Cardiology, vol. 117, no. 8, pp. 13551160, 2016.

[17] B. L. Heitmann and P. Frederiksen, "Thigh circumference and risk of heart disease and premature death: prospective cohort study," BMJ, vol. 339, article b3292, 2009.

[18] T. Rantanen, P. Era, and E. Heikkinen, "Physical activity and the changes in maximal isometric strength in men and women from the age of 75 to 80 years," Journal of the American Geriatrics Society, vol. 45, no. 12, pp. 1439-1445, 1997.

[19] I. M. Lee, E. J. Shiroma, F. Lobelo et al., "Effect of physical inactivity on major non-communicable diseases worldwide: an analysis of burden of disease and life expectancy," Lancet, vol. 380, no. 9838, pp. 219-229, 2012.

[20] H. Hamasaki, "Daily physical activity and type 2 diabetes: a review," World Journal of Diabetes, vol. 7, no. 12, pp. 243$251,2016$.

[21] B. K. Pedersen and M. A. Febbraio, "Muscles, exercise and obesity: skeletal muscle as a secretory organ," Nature Reviews Endocrinology, vol. 8, no. 8, pp. 457-465, 2012.

[22] B. P. Carson, "The potential role of contraction-induced myokines in the regulation of metabolic function for the prevention and treatment of type 2 diabetes," Front Endocrinol (Lausanne), vol. 8, p. 97, 2017.
[23] M. Ochi, K. Kohara, Y. Tabara et al., "Arterial stiffness is associated with low thigh muscle mass in middle-aged to elderly men," Atherosclerosis, vol. 212, no. 1, pp. 327-332, 2010.

[24] S. W. Lee, Y. Youm, C. O. Kim et al., "Association between skeletal muscle mass and radial augmentation index in an elderly Korean population," Archives of Gerontology and Geriatrics, vol. 59, no. 1, pp. 49-55, 2014.

[25] J. G. Ryall, J. D. Schertzer, and G. S. Lynch, "Cellular and molecular mechanisms underlying age-related skeletal muscle wasting and weakness," Biogerontology, vol. 9, no. 4, pp. 213228, 2008.

[26] B. A. Kingwell, "Large artery stiffness: implications for exercise capacity and cardiovascular risk," Clinical and Experimental Pharmacology \& Physiology, vol. 29, no. 3, pp. 214-217, 2002.

[27] I. Janssen, S. B. Heymsfield, Z. M. Wang, and R. Ross, "Skeletal muscle mass and distribution in 468 men and women aged 18-88 yr," Journal of Applied Physiology (1985), vol. 89, no. 1, pp. 81-88, 2000.

[28] L. Chen, D. R. Nelson, Y. Zhao, Z. Cui, and J. A. Johnston, "Relationship between muscle mass and muscle strength, and the impact of comorbidities: a population-based, crosssectional study of older adults in the United States," BMC Geriatrics, vol. 13, p. 74, 2013. 


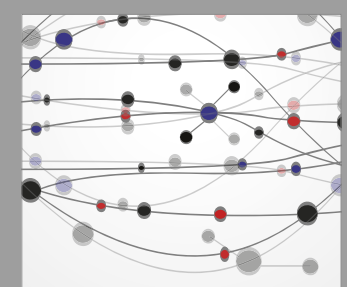

The Scientific World Journal
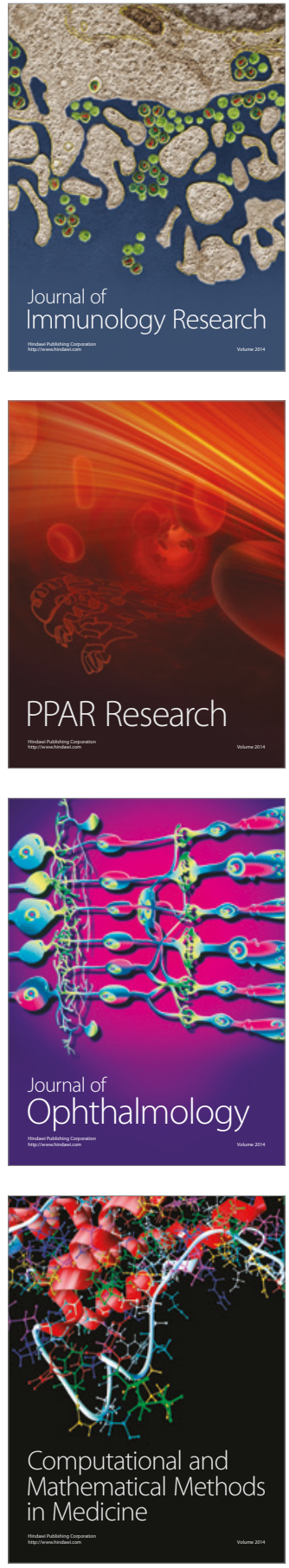

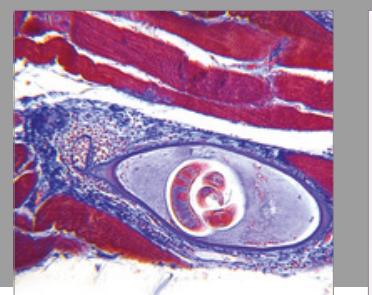

Gastroenterology Research and Practice
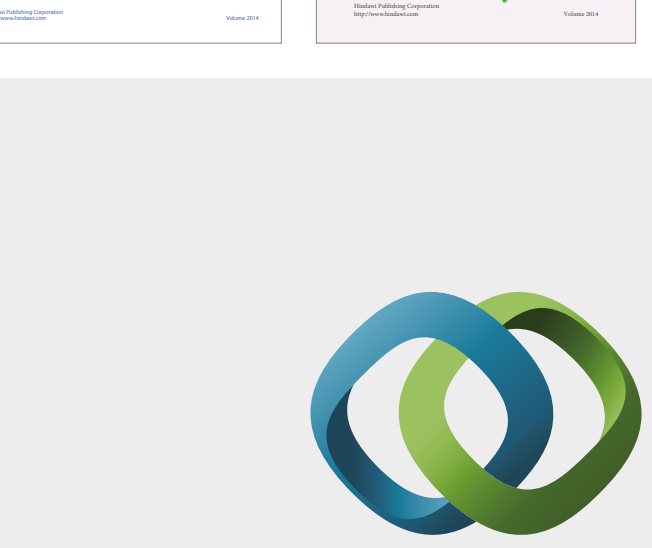

\section{Hindawi}

Submit your manuscripts at

https://www.hindawi.com
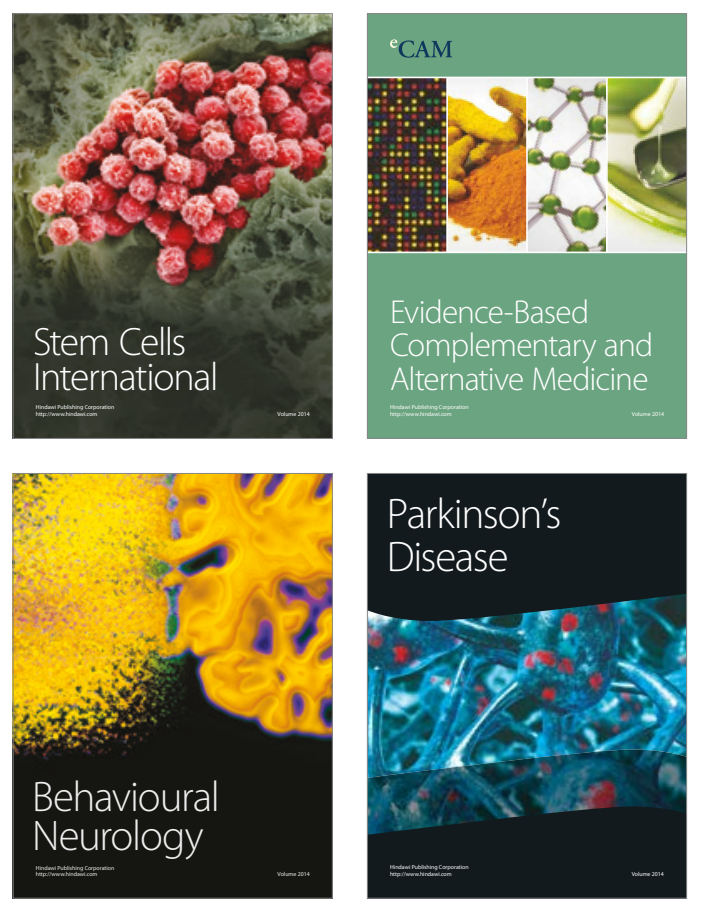
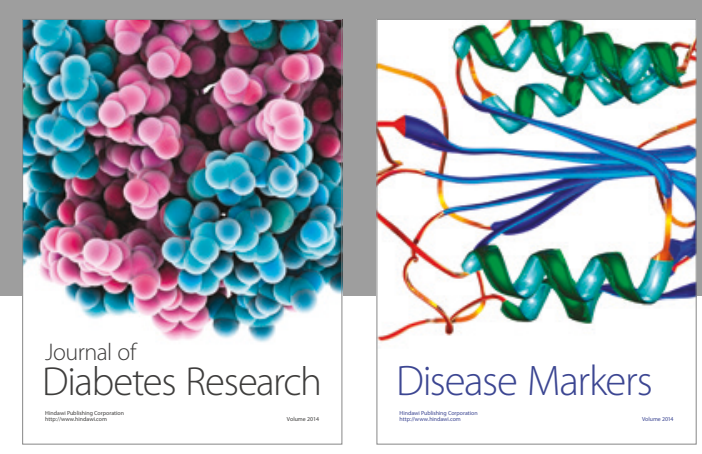

Disease Markers
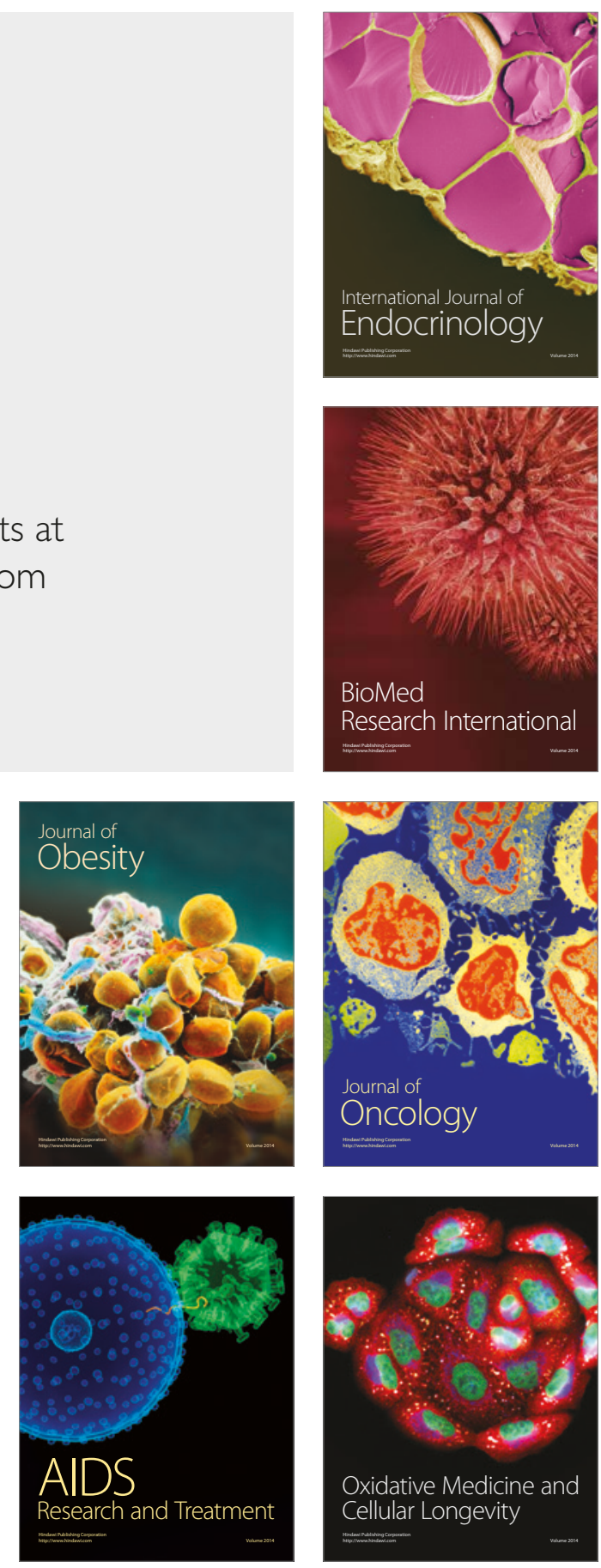